\title{
An Upper Bound on the Number of Bent Functions *
}

\author{
Vladimir N. Potapov \\ Sobolev Institute of Mathematics, Novosibirsk, Russia; email: vpotapov@math.nsc.ru
}

\begin{abstract}
The number of $n$-ary bent functions is less than $2^{3 \cdot 2^{n-3}(1+o(1))}$ as $n$ is even and $n \rightarrow \infty$.
\end{abstract}

Keywords: Boolean function, bent function, upper bound

\section{Introduction}

Let $F=\{0,1\}$. The set $F^{n}$ is called the $n$-dimensional boolean hypercube (or the boolean $n$-cube). The hypercube $F^{n}$ equipped with scalar multiplication and coordinate-wise modulo 2 addition $\oplus$ can be considered as an $n$-dimensional vector space. Functions $\phi_{x}(y)=(-1)^{\langle x, y\rangle}$ are called characters. Here $\langle x, y\rangle=x_{1} y_{1} \oplus \cdots \oplus x_{n} y_{n}$ is the inner product. Let $f$ be a function that maps from the boolean hypercube to real numbers. The Fourier transform of $g$ is defined by the formula $\widehat{g}(y)=\left(g, \phi_{y}\right)$, i.e., $\widehat{g}(y)$ are the coefficients of the expansion of $f$ in the basis of characters.

We can define the Walsh-Hadamard transform of boolean function $f: F^{n} \rightarrow F$ by the formula $W_{f}(y)=\widehat{(-1)^{b}}(y)$, i.e.,

$$
W_{f}(y)=\sum_{x \in F^{n}}(-1)^{f(x) \oplus\langle x, y\rangle}
$$

Boolean function $b$ is said to be a bent function if $W_{b}(y)= \pm 2^{n / 2}$ for all $y \in F^{n}$. It is easy to see that bent functions $b: F^{n} \rightarrow F$ exist only if $n$ is even.

Every boolean function $f$ can be represented as a polynomial $f\left(x_{1}, \ldots, x_{n}\right)=\bigoplus_{y \in F^{n}} M[f](y) x_{1}^{y_{1}} \cdots x_{n}^{y_{n}}$, where $x^{0}=1, x^{1}=x$, and $M[f]: F^{n} \rightarrow F$ is the Möbius transform of $f$. It is well known that

$$
M[f](y)=\bigoplus_{x \in \Gamma_{y}} f(x)
$$

where $\Gamma_{y}$ is the face of $F^{n}$ that contains $\overline{0}, y$ and all vectors between $\overline{0}$ and $y$. Note that $M[M[f]]=f$ for each boolean function. The degree of this polynomial is called the algebraic degree of $f$. It is easy to see that the number of boolean functions of $n$ variables with degree $\operatorname{deg} f \leq d$ is equal to $2^{\sum_{i=0}^{d}\left(\begin{array}{l}n \\ i\end{array}\right)}$.

It is well known (see [1]-3]) that the degree of a bent function $b: F^{n} \rightarrow F$ is not greater than $n / 2$. Therefore the number of bent functions is not greater than $2^{\sum_{i=0}^{n / 2}\left(\begin{array}{c}n \\ i\end{array}\right)}$. Let $N_{n}$ be the number

*The work was supported by the program of fundamental scientific researches of the SB RAS I.5.1, project No. 0314-2019-0017. 
of bent functions on $n$ variables. Then $\log N_{n} \leq 2^{n-1}+\frac{1}{2}\left(\begin{array}{c}n \\ n / 2\end{array}\right)$. In [5] and [6] there are some upper bounds of $N_{n}$. These bounds have type $\log N_{n} \leq 2^{n-1}(1+o(1))$ and they are a bit better than the trivial upper bound based on the estimation of algebraic degree. We obtain new upper bound $\log N_{n} \leq 3 \cdot 2^{n-3}(1+o(1))$. Note that Tokareva's conjecture (see [4]) of the decomposition of boolean functions into sums of bent functions implies that $\log N_{n} \geq 2^{n-2}+\frac{1}{2}\left(\begin{array}{c}n \\ n / 2\end{array}\right)$.

\section{Preliminaries}

It is well known (see [1],[7]) that

$$
\widehat{f * g}=\widehat{f} \cdot \widehat{g} \quad \text { and } \quad \widehat{(\widehat{f})}=2^{n} f
$$

where $f * g(z)=\sum_{x \in F^{n}} f(x) g(z \oplus x)$. Consequently, it holds

$$
2^{n} f * g=\widehat{\widehat{f} \cdot \widehat{g}}
$$

Let $\Gamma, \overline{0} \in \Gamma$, be a face of hypercube and let $\Gamma^{\perp}$ be the dual face, i.e., $\Gamma^{\perp}=\left\{y \in F^{n}: \forall x \in\right.$ $\Gamma,\langle x, y\rangle=0\}$. Denote by $\mathbf{1}_{S}$ an indicator function of a set $S$. If $g=\mathbf{1}_{\Gamma^{\perp}}$ then by (2) it follows

$$
f * \mathbf{1}_{\Gamma^{\perp}}=2^{-\operatorname{dim} \Gamma \widehat{\widehat{f} \cdot \mathbf{1}_{\Gamma}}}
$$

for any face $\Gamma \subset F^{n}, \overline{0} \in \Gamma$.

Lemma 1. Suppose that $f$ and $g$ are boolean functions in $n$ variables. For any face $\Gamma \subset F^{n}$ if $\left.W_{f}\right|_{\Gamma}=\left.W_{g}\right|_{\Gamma}$ then $\sum_{x \in z \oplus \Gamma^{\perp}}(-1)^{f(x)}=\sum_{x \in z \oplus \Gamma^{\perp}}(-1)^{g(x)}$ for any $z \in F^{n}$.

Proof. It follows from (33). Indeed it holds $\widehat{(-1)^{f}} \cdot \mathbf{1}_{\Gamma}=\widehat{(-1)^{g}} \cdot \mathbf{1}_{\Gamma}$ by conditions of the lemma. Then $(-1)^{f} * \mathbf{1}_{\Gamma^{\perp}}=(-1)^{g} * \mathbf{1}_{\Gamma^{\perp}}$. It is clear that $\left((-1)^{f} * \mathbf{1}_{\Gamma^{\perp}}\right)(z)=\sum_{x \in z \oplus \Gamma^{\perp}}(-1)^{f(x)}$. Hence we obtain the required conclusion.

Denote by $\operatorname{wt}(z)$ the number of units in $z \in F^{n}$. Let $B_{r}$ be a ball with radius $r$ in $F^{n}$, i.e., $B_{r}=\left\{x \in F^{n}: \operatorname{wt}(x) \leq r\right\}$.

Lemma 2. Suppose that $f$ and $g$ are $n$-ary boolean functions and $\max \{\operatorname{deg}(f), \operatorname{deg}(g)\} \leq r$. If $\left.f\right|_{B_{r}}=\left.g\right|_{B_{r}}$ then $f=g$.

Proof. By the hypothesis of the lemma we have $M[f](y)=M[g](y)=0$ if $\operatorname{wt}(y)>r$. By (11) for any $y \in F^{n}$ such that $\operatorname{wt}(y)=r+1$ we obtain

$$
\begin{aligned}
& M[f](y)=\bigoplus_{x \in \Gamma_{y}} f(x)=f(y) \oplus \bigoplus_{x \in \Gamma_{y} \cap B_{r}} f(x)= \\
= & f(y) \oplus \bigoplus_{x \in \Gamma_{y} \cap B_{r}} g(x)=M[g](y) \oplus f(y) \oplus g(y) .
\end{aligned}
$$

Therefore $f(y)=g(y)$ for any $y \in B_{r+1}$. By induction on weights wt $(y)$ of the vectors $y \in F^{n}$ we obtain that $f(y)=g(y)$ for all $y \in F^{n}$.

We will use the following property of bent functions.

Proposition 1. ([1]-[3]) Let $f: F^{n} \rightarrow F$ be a bent function, let $A: F^{n} \rightarrow F^{n}$ be a nondegenerate affine transformation and let $\ell: F^{n} \rightarrow F$ be an affine function. Then $g=f \circ A \oplus \ell$ is a bent function.

Functions $f$ and $g$ from Proposition 1 are called equivalent. It is easy to see that the cardinality of any equivalence class is not greater than $a_{n}=2^{n^{2}}(1+o(1))$. 


\section{Main result}

Theorem 1. The number of bent functions in $n$ variables is not greater than $6^{3 \cdot 2^{n-6}} 2^{\cdot 2^{n-2}(1+o(1))}$ as $n$ is even and $n \rightarrow \infty$.

Proof. Let $b$ be a bent function in $n$ variables. It is well known (see [1] -3 ) that $W_{b}=$ $2^{n / 2}(-1)^{g}$ where $g$ is a bent function too. Therefore $\operatorname{deg}(g) \leq \frac{n}{2}$. Consider a face $\Gamma, \overline{0} \in \Gamma$ and $\operatorname{dim} \Gamma=n-2$. By Lemma 2 there exist at most $T_{n}=2^{\sum_{i=0}^{n / 2}\left(\begin{array}{c}n-2 \\ i\end{array}\right)}=2^{2^{n-3}(1+o(1))}$ different functions $g^{\prime}: \Gamma \rightarrow F$ such that $g^{\prime}=\left.g\right|_{\Gamma}$ and $g$ are bent functions in $n$ variables. By Lemma 1 we obtain that $g^{\prime}$ is determined by sums $\sum_{x \in z \oplus \Gamma^{\perp}}(-1)^{b(x)}$ for all $z \in F^{n}$. It is easy to see that the sums $\sum_{x \in z \oplus \Gamma^{\perp}}(-1)^{b(x)}$ can be equal to $-4,-2,0,2$ or 4 . If a sum equals \pm 4 then $b(x)=1$ for all $x \in z \oplus \Gamma^{\perp}$ or $b(x)=0$ for all $x \in z \oplus \Gamma^{\perp}$. If it equals \pm 2 then we have four possibilities for the vector of values of $b$ on $z \oplus \Gamma^{\perp}$. At last if $\sum_{x \in z \oplus \Gamma^{\perp}}(-1)^{b(x)}=0$ then we have six possibilities for

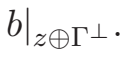

For any bent function $b$ the proportion of a 2-dimensional subspace $S$ of $F^{n}$ such that $\sum_{x \in S}(-1)^{b(x)}= \pm 2$ is equal to $\frac{1}{2}$ (for example, see [8]). Then we can find a non-degenerate linear transformation $A$ of $b$ such that the proportion of 2-dimensional faces $z \oplus \Gamma^{\perp}$ with $\sum_{x \in z \oplus \Gamma^{\perp}}(-1)^{b \circ A(x)}= \pm 2$ is not greater than $\frac{1}{2}$.

Let $\mathcal{L}$ be the set of all affine functions. If $\sum_{x \in z \oplus \Gamma^{\perp}}(-1)^{b \circ A(x)}=0$ then $\sum_{x \in z \oplus \Gamma^{\perp}}(-1)^{b \circ A(x) \oplus \ell(x)}=$ \pm 4 for $\frac{1}{4}$ of functions $\ell \in \mathcal{L}$. Therefore we can find $\ell_{0}$ with such proportion or better.

At last we note that by Lemma 2 it is necessary to recover $b$ only on $B_{n / 2}$. Suppose that $b$ is a function with the best proportion of sums $-4,-2,0,2,4$ from some equivalence class. Denote by $Q_{n}$ the minimum number of elements from $\Gamma$ that is needed for recovering $b$. It is easy to see that $Q_{n}=2^{n-3}(1+o(1))$. Since $b^{\prime}(x)=b(x) \oplus a$ is a bent function, without loss of generality, we suppose that $b$ has the best proportion of sums $-4,-2,0,2,4$ on faces which is determined by this $Q_{n}$ elements. Therefore an upper bound of the number of bent functions is $a_{n} T_{n} 4^{Q_{n} / 2} 6^{3 Q_{n} / 8}$ where $a_{n}$ is the cardinality of the largest equivalence class.

It is easy to see that $6^{3 \cdot 2^{n-6}} 2^{2^{n-2}}$ is less than $2^{3 \cdot 2^{n-3}}$.

\section{References}

[1] C. Carlet, Boolean Functions for Cryptography and Coding Theory. Cambridge University Press, 562 pages, 2020.

[2] C. Carlet and S. Mesnager, "Four decades of research on bent functions," Des. Codes Cryptogr., vol. 78(1), 2016, pp. 5-50.

[3] S. Mesnager, Bent Functions: Fundamentals and Results. Springer International Publishing Switzerland, 2016.

[4] N. Tokareva, "On the number of bent functions from iterative constructions: lower bounds and hypothesis," Adv. Math. Commun., vol. 5(4), 2011, pp. 609-621. 
[5] C. Carlet and A. Klapper, "Upper bounds on the number of resilient functions and of bent functions," Proceedings of the 23rd Symposium on Information Theory in the Benelux, Louvain-La-Neuve, Belgium. 2002.

[6] S.V. Agievich, "On the continuation to bent functions and upper bounds on their number," Prikl. Diskr. Mat. Suppl., no. 13, 2020, pp. 18-21 (in Russian).

[7] M.A. Tsfasman and S.G. Vladuts, Algebraic geometric codes. Basic notations. Mathematical Surveys and Monographs 139, Providence, RI: American Mathematical Society, 2007.

[8] V.N. Potapov and S.V. Avgustinovich, "Combinatorial designs, difference sets, and bent functions as perfect colorings of graphs and multigraphs," Siberian Math. J., vol. 61(5), 2020, pp. 867-877. 